\title{
Hippocampal overexpression of the cerebral dopamine neurotrophic factor (CDNF) impaired fear memory formation in rats
}

\author{
Tatiana Ilchibaeva \\ ICG SB RAS, Novosibirsk, Russia \\ rbicehok@mail.ru
}

\author{
Elizaveta Zolotenkova \\ ICG SB RAS, Novosibirsk, Russia \\ blanka.ardeo@yandex.ru \\ Anton Tsybko \\ ICG SB RAS, Novosibirsk, Russia \\ antoncybko@mail.ru
}

\author{
Dmitry Eremin \\ ICG SB RAS, Novosibirsk, Russia \\ dima.969696@mail.ru
}

\begin{abstract}
For the first time it was found that AAVmediated CDNF overexpression in the dorsal hippocampus of rats can impaired formation of cued fear memory which was accompanied by increase in CREB1, c-Fos and 5-HT1B receptor mRNA level.
\end{abstract}

Keywords - CDNF, AAV-mediated gene transduction, fear conditioning, CREB1, c-Fos, 5-HT receptors, $m R N A$

Motivation and aim

\section{Motivation}

CDNF is considered as a protective factor for the brain dopaminergic neurons and has recently entered clinical trials as an agent against Parkinson's disease [1]. However, the role of CDNF in the regulation of various forms of behavior outside locomotor activity remains unclear. There are some evidences on the implication of CDNF in the formation of spatial memory [2]. It is necessary to establish whether CDNF is involved in the regulation of other forms of memory, e.g. fear memory.

Aim

The aim of present study was to investigate the effect of hippocampal CDNF overexpression on the cued fear memory and neuroplasticity related genes expression in rats.

\section{Methods}

We have constructed the pAAV-Syn(0.5)-CDNF_EGFP plasmid and used AAV-mediated gene delivery to induce overexpression of CDNF in the hippocampal neurons of adult males of Wistar rats. Four weeks after stereotactic delivery of AAV-CDNF into dorsal hippocampus the fear conditioning was performed. On the training day 1 rats were placed in a fear conditioning apparatus for 3 min prior to three presentations of auditory cues ( $80 \mathrm{~dB}$ white noise, $30 \mathrm{~s}$ ), each co-terminating with a foot shock $(1.0 \mathrm{~mA}, 2 \mathrm{~s}$.) and spaced $60 \mathrm{~s}$ apart. After the last shock, the animals remained in the chambers for an additional 3 min. Cued fear memory was assessed by exposing the animals to the same context in the presence of tone presentation but without foot shocks on the day 2, 4 and 6 after training. The time spent by each rat freezing was measured. Expression of neuroplasticity-related genes (Bdnf, Creb1, cFos, Ark, Trkb, Ngfr, Htrla, Htrlb, Htr2a and Htr7) was assessed by real-time RT-PCR.
Results

We have found that CDNF overexpression impaired cued fear conditioning that was reflected in significantly low total time of freezing as well as freezing time before, during and after tone presentation on the second, fourth and sixth days after training. It should be noted that freezing in experimental and sham-operated animals was not differ on the first training day. The mRNA levels of Crebl, c-Fos and Htrlb genes were significantly increased in the hippocampus of rats with overexpression of CDNF. mRNA levels of another investigated genes were unchanged. Thus, for the first time we have demonstrated that endogenously overexpressed CDNF can negatively regulate the cued fear memory formation involving key transducers of synaptic plasticity CREB1 and cFos. 5-HT system also might be affected by CDNF.

\section{ACKNOWLEDGMENT}

The work supported by the RSF (\#19-75-00016). The cost of animal maintenance was supported by the basic research project No 0324-2019-0041-C-01.

\section{REFERENCES}

[1] Huttunen H.J., Saarma M. (2019) CDNF Protein Therapy in Parkinson's Disease. Cell Transplant. 28(4):349-366.

[2] Kemppainen S. et al. (2015) Cerebral dopamine neurotrophic factor improves long-term memory in APP/PS1 transgenic mice modeling Alzheimer's disease as well as in wild-type mice. Behav Brain Res. 291:1-11 